\title{
PLÁSTICOS EN LA AGRICULTURA: BENEFICIO Y COSTO AMBIENTAL: UNA REVISIÓN
}

\section{PLASTIC PRODUCTS IN AGRICULTURE: BENEFICE AND AMBIENT COST: A REVIEW}

\author{
Ingeborg Zenner de Polanía ${ }^{1}$, Fernando Peña Baracaldo ${ }^{2}$
}

\begin{abstract}
${ }^{1}$ I.A., M.Sc., Ph.D. Universidad de Ciencias Aplicadas y Ambientales U.D.C.A, izenner@udca.edu.co; ${ }^{2}$ I.A., M.Sc. Docente Investigador, Facultad de Ingeniería Agronómica, Universidad de Ciencias Aplicadas y Ambientales U.D.C.A, Calle 222 No. 55-37, Bogotá, DC. fepena@udca.edu.co
\end{abstract}

Rev. U.D.C.A Act. \& Div. Cient. 16(1):139 - 150, 2013

\section{RESUMEN}

El empleo de diversos elementos plásticos, tales como bolsas, cintas, coberturas, mangueras, entre otras, en la agricultura y su deshecho inadecuado, se traduce en la contaminación ambiental y visual del agro colombiano. El actual manuscrito presenta un análisis de los principales usos de estos productos, de su vida útil, de su positiva o negativa biodegradabilidad y de su posible costo ambiental, basado en recomendaciones de su manejo, descrito en la literatura, observaciones de campo y sugerencias de posibles maneras de disponer de estas "basuras", una vez que los elementos cumplan su propósito. Igualmente, se hace un análisis de un factible remplazo del "plástico" por otros elementos, menos contaminantes, pero potencialmente utilizables, para los propósitos enunciados, en bien de la producción agrícola de frutas, de la protección del suelo, del manejo de malezas, entre otras prácticas agronómicas. Se concluye que, a pesar de la amplia aceptación de la tecnología de la plasticultura, no se fundamenta en investigación autóctona que comprueba sus beneficios, sino en la adopción de conocimientos foráneos. Considerable actividad investigadora, se está realizando, sin embargo, en el país en la búsqueda de polímeros biodegradables que podrían reemplazar los sintéticos.

Palabras clave: Polímeros, contaminación, prácticas agrícolas, almidón, sostenibilidad.

\section{SUMMARY}

The use in agriculture of diverse plastic elements, such as bags, covers, hoses, between others, and the inappropriate destruction, translates into environmental and visual pollution of the Colombian field. The present manuscript presents an analysis of the main uses of these products, of their useful life, of its positive or negative bio-degradability and its potential environmental cost, based on recommendations of its management, described in the literature, field observations and suggestions of ways to dispose of this "rubbish", once the elements comply with its purpose. Also, an analysis of a possible replacement of the "plastic" by other elements, less polluting, but potentially usable for the purposes are included in the interest of the agricultural fruit production, soil protection, weed management, between other agronomic practices. We conclude that despite the wide acceptance of the plasticulture technology this is not based on indigenous research that proves its benefits, but in adopting foreign knowledge. Substantial research activity is however being performed in the country in search of biodegradable polymers that could replace the synthetic ones.

Key words: Polymers, pollution, agricultural practices, starch, sustainability.

\section{INTRODUCCIÓN}

El empleo de diferentes tipos de polímeros sintéticos en la agricultura es una tecnología emergente que ha permitido convertir tierras aparentemente improductivas en explotaciones agrícolas productivas y, en algunos casos, incrementar la calidad de frutas y de hortalizas. Tiene múltiples aplicaciones, que se concentran en su uso en invernaderos, en túneles, en microtúneles, en alcochado o mulching, en mallas para sombrío y en embolse (Macías et al. 2011; Stavisky, 2010; Espi et al. 2006). La plasticultura, el uso de plásticos en la agricultura, como lo definen Delgado et al. (2011), se introdujo en los países desarrollados en la mitad del siglo pasado, mientras que los países en vía de desarrollo adoptaron esta tecnología, en forma profusa, en los años noventa. De acuerdo a Espi et al. (2006), los materiales más emplea- 
dos para invernaderos y túneles corresponden a polietileno de baja densidad y copolímeros de acetato de vinil etileno o acrilato butil etileno y de polietileno de baja densidad lineal para el acolchado.

La tecnología emplea estos elementos plásticos de corta vida útil que, cumplidos su función, se convierten, al no ser reciclado, en basura contaminante. La mayoría de estos productos son de baja biodegradabilidad (alta recalcitrancia), generando elevadas cantidades de residuos (Meneses et al. 2007). Permanecen, a la vista causando, fuera de la polución ambiental, una marcada contaminación visual. De este aspecto negativo, se percata el habitante de las ciudades al pasear por el campo en busca de esparcimiento o, como ocurre en muchos casos, compra de frutas y de verduras, empacadas en bolsas plásticas, que luego terminan en el borde de las carreteras. A menudo, se observan también invernaderos improductivos abandonados y la dispersión del plástico de cobertura por el viento (Foto 1a).

El polietileno agrupa una familia numerosa de polímeros de distintas estructuras moleculares, que confieren distintas características. Tenemos: polietileno de Alta Densidad (PEAD), Polietileno de Baja Densidad (PEBD) y Polietileno Lineal de Baja Densidad (PELBD) (butano, hexeno, octeno) (Espi et al. 2006; González-Viñas \& Mancini, 2003).

Aquellos investigadores que están encaminando sus trabajos a la búsqueda de alternativas poliméricas biológicas para reemplazar los sólidos plásticos consideran, precisamente, que estos materiales son los contaminantes más comunes, a nivel mundial (Baraldi de Pauli et al. 2001; Medina \& Salas, 2003). La preocupación acerca de los contaminantes plásticos ha llegado a que inclusive, se diseñen y evalúen cadenas de desechos agrícolas plásticos, desde la generación hasta la disposición, con fines de recuperación de energía (Hiskakis et al. 2008).

La era "plástica", se inició en Colombia en los años 70-80, con la construcción de los mal llamados "invernaderos", para el cultivo de flores, inicialmente, el clavel, con la función básica de proporcionar condiciones climáticas más favorables que las existentes externamente, para acelerar el desarrollo de la flor y programar la cosecha destinada a la exportación. Tanto los invernaderos como los túneles y microtúneles facilitan también el manejo de las heladas con quemadores y riego. Estas estructuras cubiertas con "plástico" son también utilizados para hortalizas, como es el caso de tomate, que en Colombia se cultiva en aproximadamente 3800ha, bajo estas condiciones (FAO, 2007), en el Brasil, para pimentón (Scivittaro et al. 1999) y en México, para la producción de plántulas de ají, para disminuir el impacto de las enfermedades en los semilleros a aire libre (Macías et al. 2011).
Mulching o cobertura plástica del suelo tiene la finalidad de evitar el crecimiento de malezas, disminuir la evaporación del agua de riego mejorando la retención de humedad y evitar el contacto de los frutos con el suelo; su mayor aplicación se tiene en la actualidad para el cultivo de fresa (Santos \& Obregón, 2009; Yuri et al. 2012); otras especies vegetales que son cultivadas bajo estas condiciones son el melón (Medeiros et al. 2006; Silva et al. 2005). Una investigación fue ejecutada por Arruda et al. (2005) en el algodonero en Brasil, empleando cobertura para el control del coquito Cyperus rotundus, en cultivos con y sin riego. Establecieron que la plasticultura para el control de malezas es solamente económico, en un cultivo que depende de las lluvias.

Los filmes para acolchados constituyen la segunda aplicación en importancia (luego de invernaderos), por el volumen de los plásticos en las aplicaciones agrícolas; la superficie mundial bajo esta modalidad es de 4.530.000ha. Detallando por países, se destaca China, con 2.000.000ha; Japón, con 150.000ha y, Francia y España, con 100.000ha, cada uno. En Latinoamérica esta aplicación se ha desarrollado principalmente en Centroamérica y en México, superando este último país las 9000ha (Thompson et al. 2009).

Mallas para sombrío: Normalmente de color negro y de diversos mesh y calibre, cubren diversos cultivos, ante todo flores y las hortalizas, en la zona cafetera del país. Estas mallas evitan la incidencia directa de los rayos solares y modifica el microclima. En el Brasil, su empleo se ha generalizado en algunas áreas en el cultivo de la vid, con el propósito de disminuir la incidencia de enfermedades y promover un aumento en el desarrollo de la planta y del rendimiento (Chavarria \& Santos, 2009; Chavarria et al. 2007).

Embolse: El uso masivo en la agricultura del embolse de frutas, se inició a principios de los años noventa, para la protección de los racimos de plátano y en plantaciones, cuya producción tenía la exportación, como fin principal (Belalcazar et al. 1991). Esta práctica ya estaba siendo utilizada con éxito en Costa Rica (Vargas et al. 2010), con anterioridad en el Brasil (Rodrigues et al. 2001; Moreira (1987) y en Australia, donde ha sido de uso común desde los años cincuenta (Berrill, 1956; Robinson \& Nel, 1984).

Fuera de la contaminación con los desechos parecen existir pocas desventajas de la plasticultura. Una revisión acerca del manejo de enfermedades fue realizado por Vida et al. (2004) y concluyeron que las condiciones climáticas bajo techo pueden incrementar el impacto de algunos problemas bióticos y abióticos y, además, que enfermedades secundarias se pueden convertir en destructivas. Observaciones similares realizaron los autores de esta revisión en relación a plagas insectiles, como por ejemplo, la mosca blanca de los inver- 
naderos, cuyas poblaciones en tomate son alarmantes. Otra desventaja del cambio de las condiciones climáticas representa la factibilidad que insectos de otros pisos térmicos se adapten y causen daño económico a los cultivos bajo techo.

El objetivo de la presente revisión no fue solamente presentar el panorama, sino también advertir acerca de la prácticamente nula investigación sobre el tema en el país y alertar a la sociedad rural y urbana sobre el daño que se está causando a las futuras generaciones. Además, examinar las posibilidades de mermar la contaminación, evaluando y aplicando alternativas ya existentes y viables de reemplazar a los polímeros sintéticos y disponer de estas "basuras", teniendo en cuenta que en la gran mayoría de las zonas rurales del país no existe el servicio de recolección de basuras.

\section{MATERIALES Y MÉTODOS}

Esta revisión, se basó en el análisis de la literatura acerca de la plasticultura en, ante todo Centro y Sur América; se centró en especies vegetales, cuya producción en Colombia, se desarrolla con la ayuda de plásticos, como son plátano y banano, fresa y lechuga. Igualmente, se examinaron lo pertinente a su vida útil, a la legislación existente sobre su disposición o reciclaje, para poder recomendar algunas prácticas que disminuyan, tanto la contaminación ambiental como la visual. También, se incluyen algunas experiencias de los autores en el uso de plásticos y observaciones de campo.

Se tuvo en cuenta que el campo de los materiales plásticos es inmenso y de una movilidad enorme. Por otra parte, ningún plástico, en su presentación en el mercado, es un material muy definido, pues la combinación de distintas resinas y la incorporación de distintos aditivos: plastificantes, antioxidantes, colorantes y otros, le confieren propiedades muy distintas.

\section{RESULTADOS Y DISCUSIÓN}

Embolse de racimos de banano y plátano: Corresponde a la práctica de colocar una bolsa de polietileno al racimo en formación con destino a exportación, para protegerlo de factores bióticos y abióticos, que demeritan su calidad. Este procedimiento está ampliamente difundido en las plantaciones de banano de Centro y Sur-américa; igualmente, está implementado para la protección de plátano en el departamento del Quindío. Las bolsas plásticas perforados del color azul (Foto $1 \mathrm{~b}$ ), con una densidad de 1,0gmL, impregnados con un insecticida son las más recomendadas (Lima, 2002). Especificaciones, establecidas en el Brasil referente a las características físicas de las bolsas, se encuentran en el tratado "O cultivo da Bananeira" (Trinidade et al. 2004); se menciona que el embolse aumenta el crecimiento de los frutos, evita el ataque de plagas, mejora la cualidad del fruto y protege los frutos del efecto abrasivo de los fungicidas utilizados contra la Sigatoka.

El incremento de la producción a raíz del embolse no se puede generalizar. Moreira (2008) determinó que el beneficio depende de los cultivares del banano, mientras que Costa et al. (2002) no encontraron diferencias entre el tamaño y la calidad del racimo. Rodrigues et al. (2001) tampoco hallaron diferencias significativas en el peso de los racimos y el peso de las manos, pero sí una reducción en el tiempo de cosecha de 12 días. Barrera et al. (2007), en la zona de Tierralta (Córdoba, Colombia), no obtuvieron una mayor producción con ésta y la práctica de desmane, pero sí un producto limpio y exportable. En esta zona y en el Urabá colombiano, los insectos que afectan los racimos y cuyo daño ocasiona el rechazo de las manos son la abejita Trigona, al cucarrón Colaspis y a los trips. Contra estas plagas y el daño causado por pájaros y por murciélagos está destinada la impregnación de las bolsas o fundas con productos químicos. Así, al disponer de manera inadecuada la funda, la contaminación aumenta aún más. El insecticida más empleado es el clorpirifos, normalmente incluido en bolsas de baja densidad. También, se han empleado piretroides, como la bifentrina, tanto en bolsas de baja como de alta densidad (Gómez \& Romero, 2002).

Tanto en el Quindío como en la zona bananera del Magdalena, el color azul de las bolsas es predominante (Foto 1a); este color permite la transmisión de calor, a la vez, que reduce el daño que puede causar la radiación solar (Robinson, 1996). La evaluación realizada por Vargas-Calvo \& Valle-Ruiz (2011) de dos tipos de fundas, una de color azul (Azul Santa Lucía) y la otra transparente foto-selectiva, ambas impregnadas con bifentrina, no mostró diferencias significativas. Tampoco se habían encontrado en Costa Rica diferencias entre los colores azul, verde y rojo y una densidad alta y baja de las bolsas de polietileno (Vargas et al. 2010).

La investigación relacionada con el embolse de los racimos de plátano y de banano es amplia y numerosa, ante todo en Australia, Costa Rica y Brasil; sin embargo, ninguno de los trabajos analizados presenta el aspecto de la contaminación y reciclaje de estas fundas. En el país existen numerosas fábricas de plásticos, dos de ellas de dedicación a los implementos de polietileno para la industria bananera. C.I. Banacol S.A, localizado en Turbo, Urabá antioqueño, produce todos los insumos plásticos para la industria bananera, desde los materiales de embolse hasta los productos de empaque (Banacol, s.f.). Igualmente, se responsabiliza de las actividades de recolección de las bolsas tratadas con clorpirifos y de entrega para la disposición final de estas bolsas a empresas acopiadoras y recicladoras en los departamentos de Antioquia, Magdalena y Quindío. Este compromiso, entre otros, está estipulado en la Resolución número (0272) 16 de febrero 2009, en la cual, llama la atención el siguiente 
a.

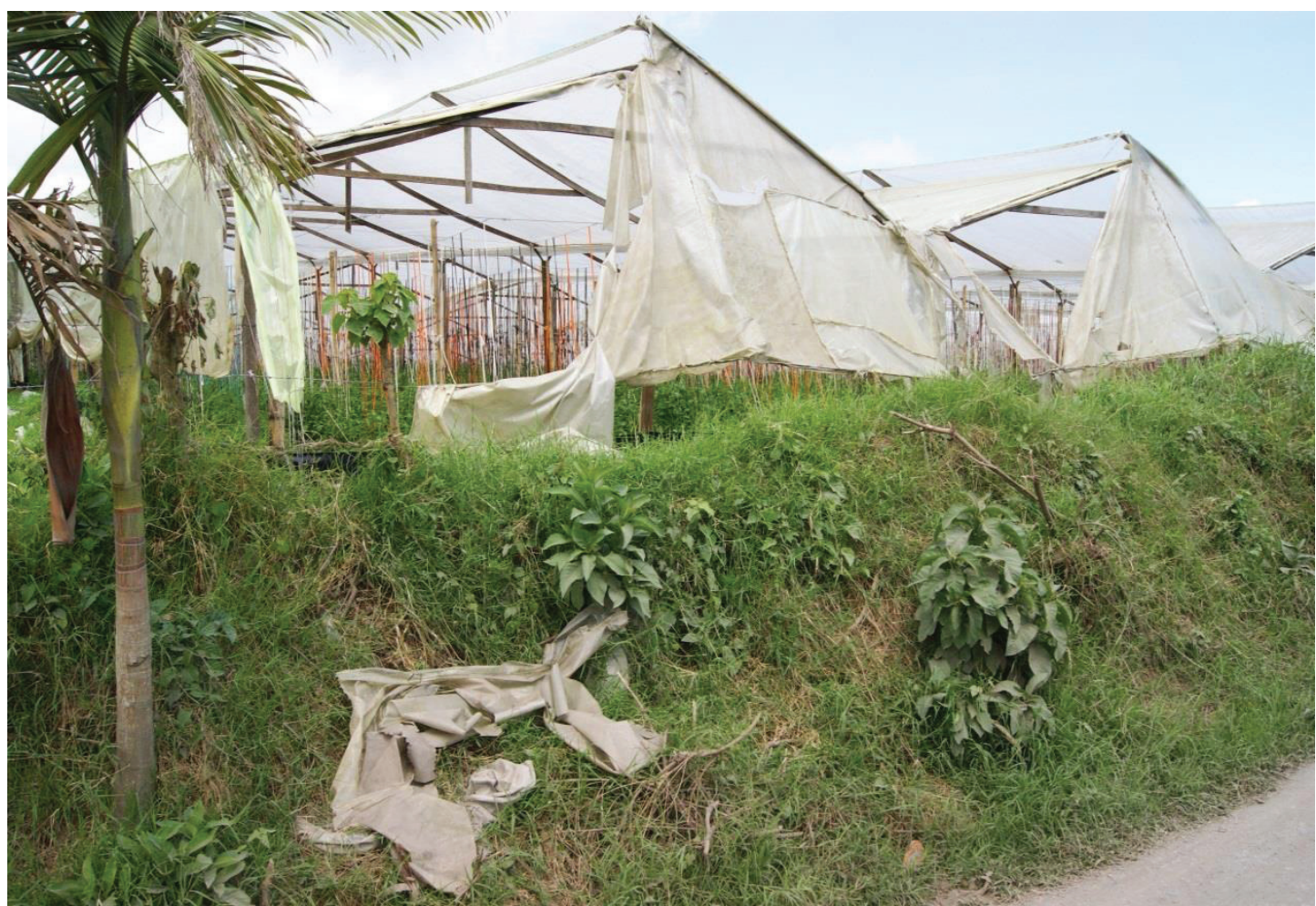

b.

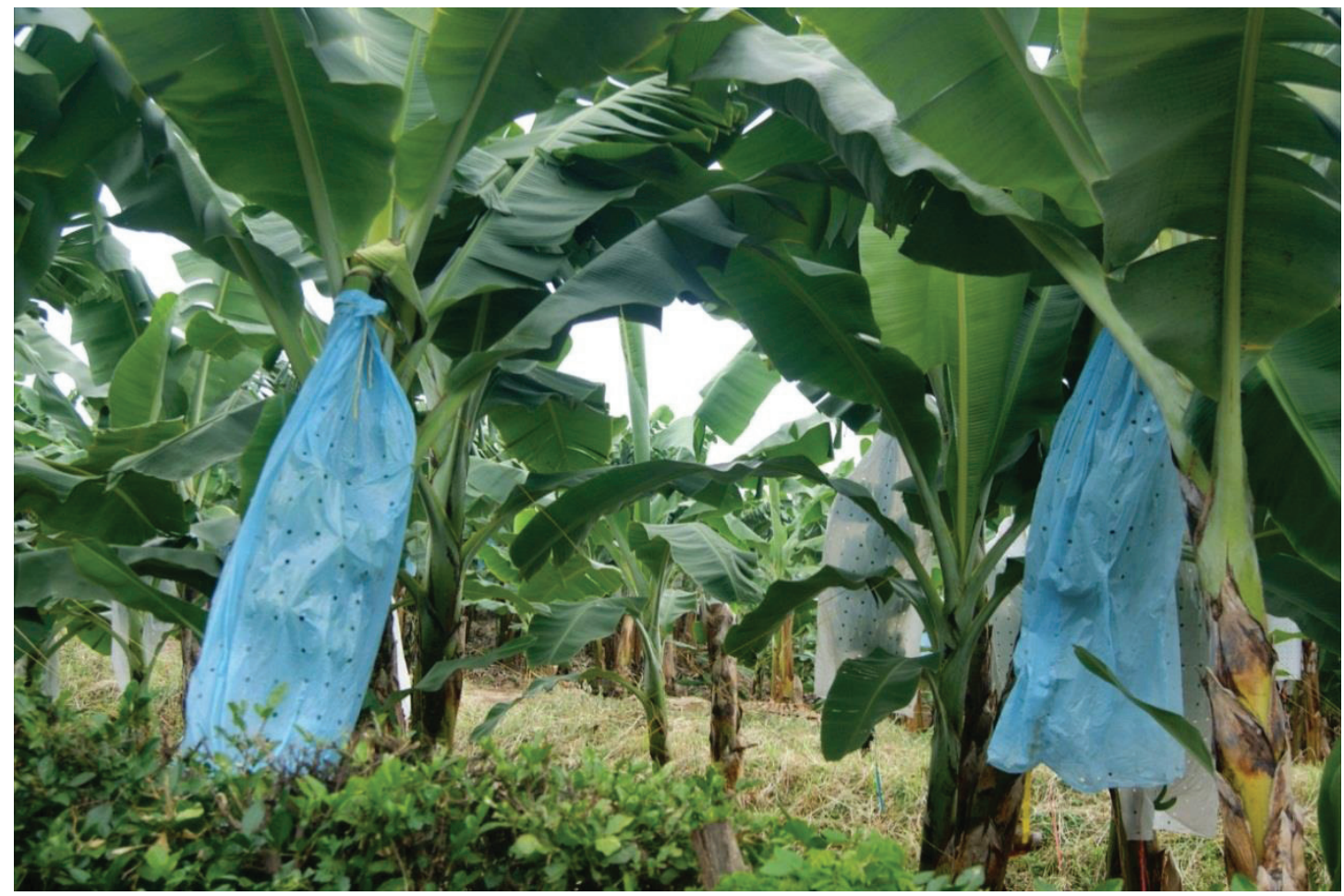

Foto 1. a: Este invernadero en el que se producía tomate fue abandonado al término de la cosecha principal y por el bajo precio de la hortaliza en CORABASTOS; b. Turistas, no familiarizados con el embolse de racimos de banano que viajan por la vía Santa Marta - Riohacha, se asombran de esta práctica. 
aparte: "Las comercializadoras en cumplimiento de normas internacionales y los planes de manejo ambiental registrados ante las diferentes corporaciones, exigirán a las fincas entregar adecuadamente la bolsa tratada poscosecha a las autorizados por C.I. Banacol S.A. Estos hacen una separación manual, secan, prensan, embalan, aglutinan y venden los reciclables": Se pregunta: ¿con estas actividades se soluciona el problema de la contaminación? Los "reciclables" siguen impregnados del insecticida clorpirifos y al convertirlos en, por ejemplo, mangueras negras de polietileno o causar contaminación del aire al incinerar los empaques de Pyritilene (clorpirifos) en una cementera de Nobsa (Boyacá), ċse puede asegurar la ausencia de contaminación?

Olefinas (s.f.) produce "fundas para embolsado", impregnadas ya sea con clorpirifos o con bifentrina. La empresa presenta un listado de recomendaciones para el cuidado y el almacenaje de sus bolsas bananeras, entre los cuales, se pueden mencionar: no comer, frotarse los ojos, ni fumar mientras embolsa; no empacar comida en este material; lavarse las manos con agua y jabón después de terminada la labor y antes de fumar o comer y evitar que los niños jueguen con este material. No figura entre las recomendaciones el cómo disponer o reciclar las bolsas ya no reutilizables.

Desde el punto de vista salud humana y daño al medio ambiente existe la resolución 637 de 2004, que hace referencia al decreto 1180 de 2003, que asigna a las Corporaciones Autónomas Regionales, entre otras obligaciones, otorgar licencia para "la construcción y operación de instalaciones cuyo objetivo sea el almacenamiento, tratamiento, aprovechamiento, recuperación y/o disposición final de residuos o desechos peligrosos...". Entre los residuos peligrosos figuran las bolsas plásticas utilizadas para la protección de racimos de plátano y de banano.

Cobertura, acolchado o mulching en fresa y lechuga: Se trata de extender sobre el suelo un material plástico, generalmente polietileno de color negro o blanco, con el fin de manejar malezas, evitar la evaporación del agua del suelo, impedir que los frutos estén en contacto con el suelo y así protegerlos de posibles pudriciones (Foto 2 a). Menciona Sanz (2012), que el plástico negro es el más empleado en España, que evita el desarrollo de malas hierbas por la barrera que suponen a la radiación luminosa, pero su influencia sobre la precocidad y el rendimiento es escasa.

Se utilizan diferentes tipos de plástico; en cuanto al color, varía dependiendo de las necesidades del cultivo y la región, cada uno de ellos posee determinadas características, que dan lugar a efectos diferentes sobre los cultivos. Es preciso que el agricultor antes de utilizarlos conozca los efectos de cada uno para tomar las decisiones más correctas, de acuerdo al cultivo que va a establecer y las condiciones climáti- cas de la época y región en que se encuentra (Medina et al. 2006). Los productores enfrentan serios problemas cuando el tipo y el color del plástico empleado no es el correcto o cuando se emplea en una zona y época donde los efectos climatológicos no actúan favorablemente en combinación con el color del acolchado empleado: Estos se ven fuertemente modificados por los diferentes colores, provocando efectos impredecibles pudiendo ser favorables o desfavorables para el cultivo, cuando no se tiene el conocimiento necesario (Medina et al. 2006).

La radiación reflejada, absorbida y trasmitida por los diferentes acolchados determina, en gran medida, la temperatura que se generan en el suelo y el efecto positivo y negativo de esta temperatura sobre el desarrollo y el rendimiento de las plantas (Oriolani et al. 1979; Thompson et al. 2009; Vargas et al. 2010). Los mismos autores designan los colores, como: Cristal, natural o transparente, que es el polietileno sin ningún tipo de pigmento ni aditivos y se usa principalmente para elevar la temperatura del suelo; Negro, presenta la menor reflexión (9\%) y es el que más se calienta pudiendo causar quemaduras en aquellas estructuras de la planta en contacto con el mulch; asegura un perfecto control de malezas a menor costo que los otros materiales verdes, blanco/negro, plata/negro; Blanco/Negro, asegura un perfecto control de malezas, se calienta menos que el negro, porque la coloración blanca refleja parte de la radiación, aumenta la radiación fotosintética que llega a la planta; Plata/Negro, permite un perfecto control de malezas, mientras que la reflexión del plata repele insectos protegiendo la planta, también disminuye la temperatura de suelo, aumenta la radiación fotosintética; Verde traslucido, ofrece un control adecuado de malezas permitiendo el calentamiento del suelo y, finalmente, Naranja/Marrón, que permite el paso del calor durante el día, con cierta opacidad para prevenir las malezas, reduciendo la pérdida de calor durante la noche.

El tratado más completo sobre este tema lo presentan Santos \& Obregón (2009), en un boletín sobre el cultivo de la fresa. Resaltan las ventajas del acolchado en comparación con la producción en suelo desnudo. Indican que incrementa el desarrollo del cultivo y los rendimientos y permite adelantar la cosecha; inhibe el crecimiento de malezas, actuando como barrera física e interceptando la radiación solar; mejora la retención de humedad, lo que permite economizar agua y reduce la pudrición de la fruta, actuando como una barrera física protectora entre el suelo y la fruta; mantiene limpia la fruta. Entre las desventajas resaltan el "retiro y descarte", considerándolo el mayor problema, ya que la mayoría de los acolchados no son biodegradables y deben ser retirados una vez cumplidos su cometido. Indican que "el descarte presenta un problema severo debido al volumen de la basura generado". El mismo problema se visualiza en España, como lo describe Sanz (2012); las coberturas de polietileno se con- 
a.

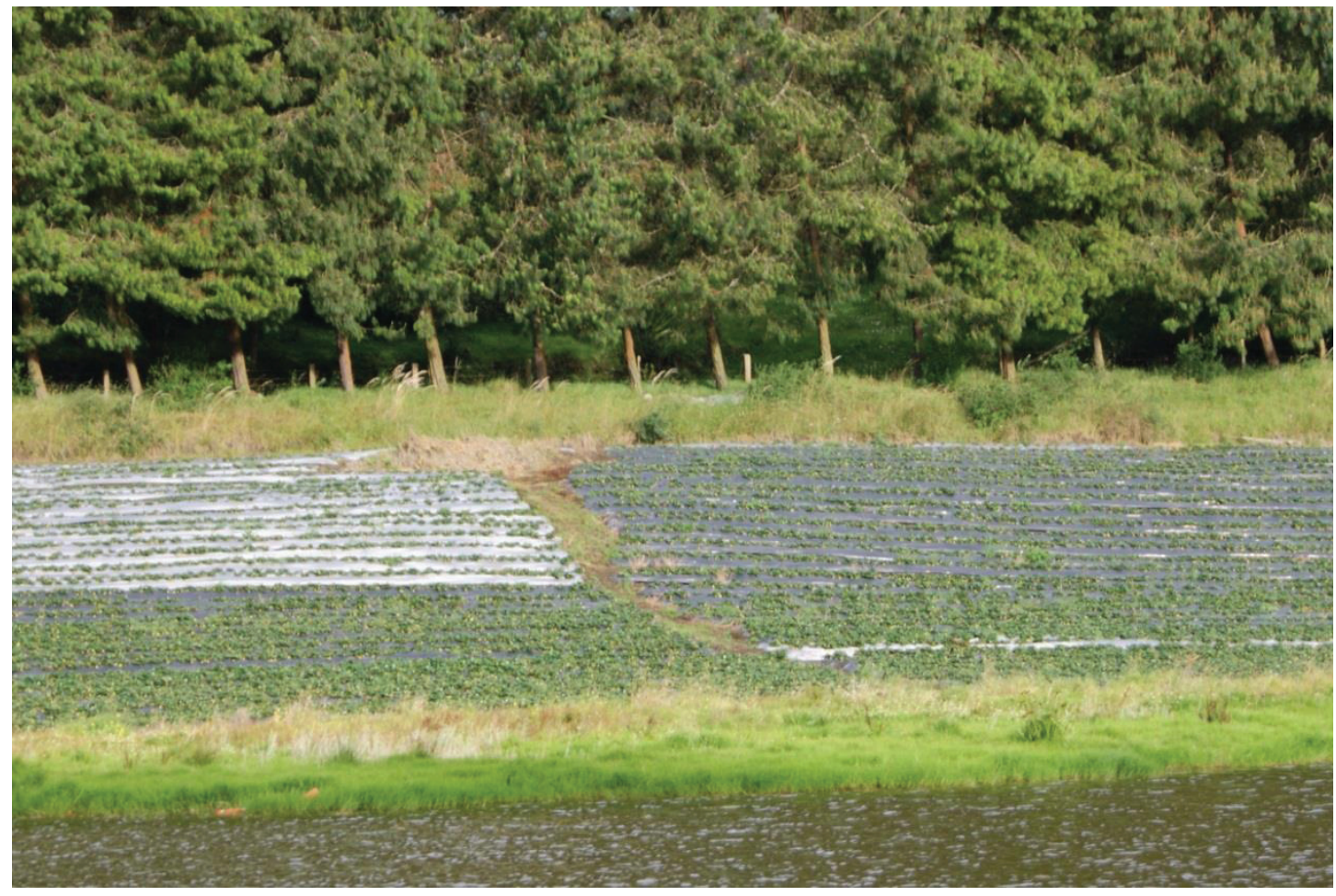

b.

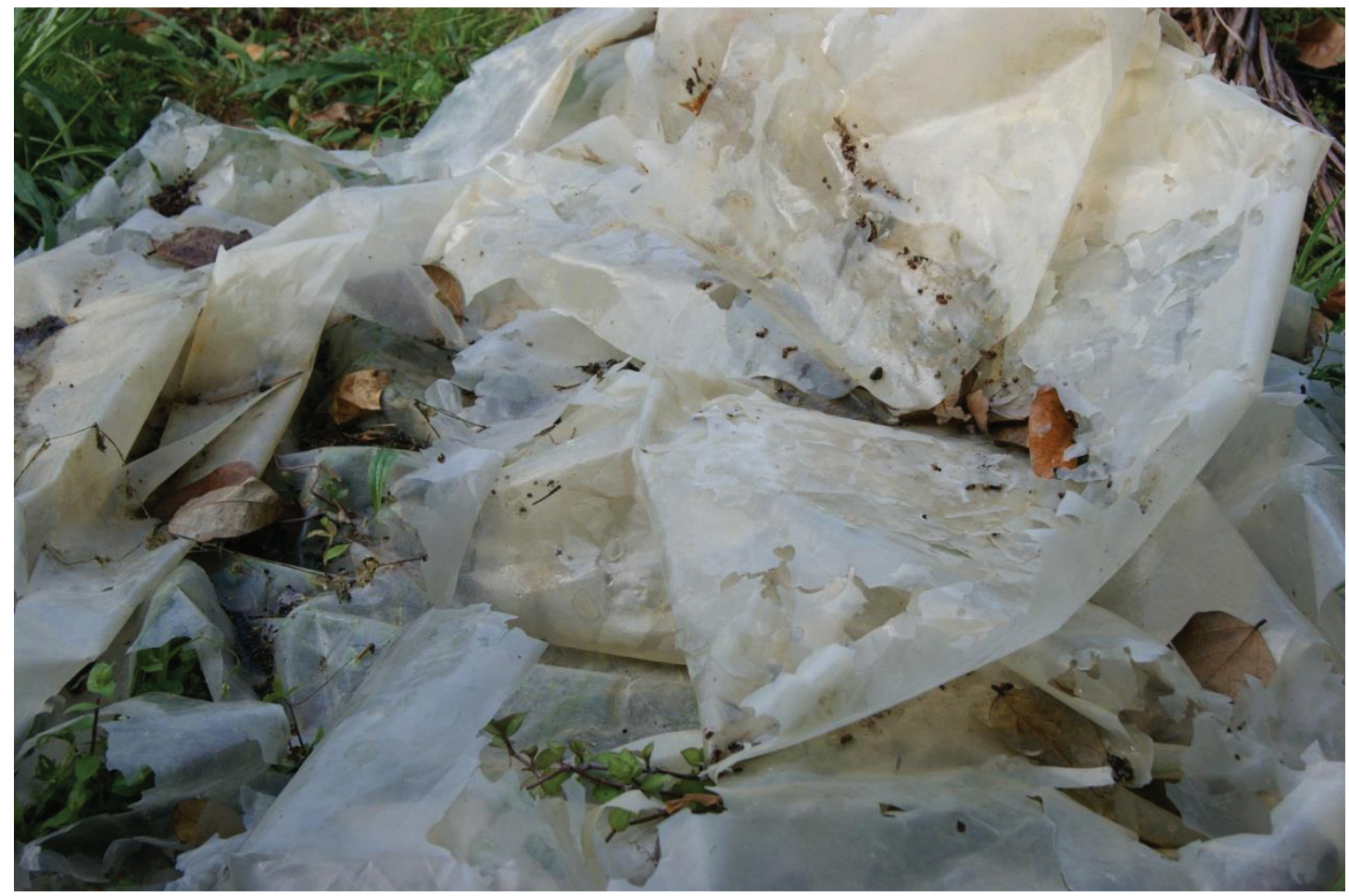

Foto 2. a. Cobertura de polietileno incoloro y negro en un cultivo de fresa en Cundinamarca; b. La degradación de un plástico, calibre 7, al cabo de un año a la intemperie es prácticamente nula. 
vierten en un residuo que tiene que ser retirado y enviado a una empresa autorizada para su destrucción o reciclaje.

Existen investigaciones que han cuantificado el ahorro de agua logrado con el uso de acolchado de polietileno, tal es el caso de Renquist et al. (1982), quienes señalan que al cultivar fresa con acolchado de polietileno en verano, se requiere un tercio del agua en comparación a la que necesita cuando es cultivada sin acolchado.

En relación del color del polietileno no existen resultados coincidentes. En la Sabana de Bogotá, se usa con mayor frecuencia el color negro, pero también se observa el color blanco (Foto 2a). Comparando, en Israel, polietileno transparente con el de color negro, Converse (1981) obtuvo un 10 a $15 \%$ de aumento en rendimiento en fresa de invierno con el uso de polietileno transparente. Igualmente, Bringhurst \& Voth (1990) tuvieron como resultado que el acolchado de polietileno transparente es una de las técnicas más recomendables para mejorar la producción de fresas en California durante la estación fría, encontraron que este tipo de polietileno es de mayor provecho en inviernos más fríos, ya que incrementa, considerablemente, la temperatura del suelo. ¿Valdría la pena evaluar, en zonas del altiplano Cundiboyacense, donde se presentan heladas, el uso del plástico transparente para disminuir el efecto de las bajas temperaturas, al aumentar aquellas del suelo?

Lechuga: Tal vez la hortaliza en la que se han realizado el mayor número de experimentos con el uso de acolchonado ha sido la lechuga, aunque en el país no se ha incursionado en el uso del mulch, para este especie vegetal. Las investigaciones realizadas en otros países, la mayoría de climas templados son relevantes y los resultados obtenidos se deberían evaluar en, por ejemplo, en el altiplano Cundiboyacense y en Nariño.

No solamente se han trabajado con acolchonados plásticos, sino también orgánicos, representados, por ejemplo, heno, cascarilla de arroz y otros productos biodegradables, incorporables al suelo. Este tipo de mulch reduce también la temperatura del suelo, mantiene la humedad y aumenta el rendimiento en comparación con el suelo descubierto, significativamente mayor que en suelo descubierto (Adentuji, 1993). Sería recomendable para su uso en zonas áridas, donde, además, los suelos, por lo general, son carentes de materia orgánica.

Igual que en fresa, existen resultados relacionados en lechuga con el color de la cobertura. El efecto de papel negro y café y polietileno negro como alcochado fue investigado por Eichin \& Deiser (1991), quienes determinaron que el desarrollo de la hortaliza no fue afectado; lograron cabezas limpias de mayor valor comercial, por la disminución de pu- drición de las hojas externas; también hubo un aceptable manejo de malezas. Schrevens et al. (2005), con un mulch de polietileno transparente, demostraron que las más altas temperaturas de suelo ocasionaron una mayor precocidad, traducida en ocho días respecto al testigo; también registraron altas temperatura de suelo bajo acolchado naranja y negro; mientras el coextruido blanco/negro presentó las más bajas temperaturas. Los mismos autores indican que la calidad de la lechuga fue mejorada con el uso de acolchados de color naranja, transparente, negro y gris humo, destacándose el tratamiento con acolchado naranja, que superó la calidad obtenida en el testigo y con acolchado blanco y coextruido blanco/negro.

También existen unos pocos trabajos referentes al efecto de la fertilización, con y sin acolchonado. En lechuga romana, se determinó un mayor nivel de nitrato en suelos con acolchado que en los descubiertos (Al-Assir et al. 1992); concluyeron los autores en climas moderados y suelos con adecuado nivel de nitrógeno, este tipo de lechuga no responde al uso de polietileno transparente. La producción de lechuga bajo techo se vio favorecida con el empleo del acolchado de polietileno, ya que el porcentaje de colonización de raíces por micorrizas vesículo arbusculares aumentó considerablemente, disminuyéndose la necesidad de fertilización potásica (Contreras et al. 1993).

Plástico biodegradable: Stevens (2001) inicia el primer capítulo de su libro "Plásticos Verdes: Una Introducción a la Nueva Ciencia de Plásticos Biodegradables", señalando que ningún producto sobre la tierra ha sido tan altamente valorado por su utilidad, pero a la vez considerado tan maligno como el plástico. Ilustra sobre el futuro de los plásticos biodegradables a base de materiales renovables, biodegradables.

En España, indica Sanz (2012), el Centro Tecnológico de la Agroindustria (Adesva), se dedica al desarrollo de plásticos a base de almidón de maíz para el acolchado de fresa. Estas investigaciones están siendo realizados en el marco del proyecto Agrobiofilm, para evitar el uso del tradicional, muy contaminante, plástico negro de polietilieno. La comparación en campo entre el plástico elaborado con almidón y el plástico sintético mostró la ausencia de diferencias significativas entre los materiales en la producción de fresa. El mulch, al terminar la cosecha, sería triturado e incorporado al suelo. En Colombia, la investigación relacionada con la obtención de polímeros biodegradables se ha concentrado en la utilización de almidón de yuca (Ruiz Aviles, 2006; Meneses et al. 2007; Ruiz et al. 2009). Hasta donde se logró determinar, por el momento, los estudios se han centrado en la evaluación de la biodegradabilidad bajos diversas condiciones, entre las cuales, figuran la simulación de un relleno sanitario. Todavía no existen, aparentemente, investigaciones relacionadas con su empleo en, por ejemplo, la fabricación de fundas para la 
protección de los racimos de musáceas o el acolchado de fresa u otros usos agrícolas.

Hasta tanto no evolucione la obtención y el uso práctico de plásticos a base de almidón procedente de diversas especies vegetales, se seguirá en la búsqueda de un aceptable reciclaje o reutilización del polietileno sintético, previa concientización de los usuarios del polímero. Aquí cabe lo que se puede observar (Foto 2b): después de un año la "degradación" de un plástico colocado a la intemperie, solamente causó la cristalización parcial; el material sigue volviéndose pedazos que son distribuidos por el viento.

\section{Consideraciones y observaciones adicionales:}

En su mayoría, el plástico proviene del petróleo y se estima que entre un 5 y $7 \%$ de la producción mundial de este recurso es destinado a la producción de este material. Para producir un kilo de plástico se requieren dos kilos de petróleo (ACRR et al. 2004). El plástico es un material inorgánico que tiene alta durabilidad. Se calcula que puede tardar entre 100 y 1000 años para degradarse, dependiendo del tipo de plástico. Hay tres grandes grupos en los que se clasifican los plásticos (González-Viñas \& Mancini, 2003): los termoestables o termo rígidos, se moldean con calor y presión y una vez están fríos adquieren una forma y no pueden volver a ser moldeados; son difíciles de reciclar, ya que para hacerlo se requiere la destrucción de su estructura molecular para poder fundirlos y esto hace que se alteren las propiedades originales, es decir, en vez de fundirse se queman cuando la temperatura aumenta. Los elastómeros presentan una gran elasticidad, tienen una gran resistencia a todo tipo de esfuerzos (tracción, compresión, torsión y flexión), se deforman cuando son sometidos a un esfuerzo, pero recuperan su forma original al dejar de ejercerse la fuerza; no toleran bien el calor, lo que dificulta su reciclado al degradarse con temperaturas no muy altas. Los termoplásticos, se derriten con el calor y se endurecen cuando se enfrían; pueden ser reciclados fácilmente, ya que mantienen sus propiedades plásticas y pueden recalentarse y formar otros objetos; sin embargo, van perdiendo propiedades por lo que no pueden ser reciclados más de 5 o 7 veces. Dentro de este grupo entran una gran variedad de termoplásticos.

Como lo plantea Leff (2003), los impactos no pueden ser valorados de acuerdo a criterios económicos exclusivamente; no obstante, existe un desconocimiento de la resilencia, regeneración y recuperación de los ecosistemas afectados por los residuos plásticos. A nivel mundial, el principal impacto ambiental de estos residuos es la contaminación de los océanos. Se han encontrado cantidades substanciales contaminando los hábitats marinos, desde costas remotas inhabitadas hasta costas altamente pobladas y áreas profundas del océano (Thompson et al. 2009; Barnes et al. 2009).
En el caso de los rellenos sanitarios, los aditivos y los elementos constitutivos pueden ser liberados e introducidos al ambiente (Teuten et al. 2009). No todos los residuos plásticos son perceptibles a la vista, pues se desintegran por las condiciones de exposición al sol y lo que se detecta son los fragmentos de este material (Thomson et al. 2004).

El PVC también ha sido cuestionado por sus impactos en la salud humana y en el ambiente. Para obtener el cloruro de polivinilo, se requiere gas de cloro, usando el $40 \%$ de la producción mundial del mismo, unos 16 millones de toneladas. El PVC es el responsable del mayor volumen de producción de organoclorados, una clase de químicos que están siendo analizados desde la última década, por sus severos riesgos (Thornton, 2002).

Se han desarrollado, a nivel mundial, diferentes técnicas para reciclar los residuos plásticos. Aunque, se debe recordar que el reciclaje de plásticos tiene restricciones, dentro de las limitaciones, se encuentra que no todos los plásticos son reciclables. No solamente los elastómeros y los termorígidos presentan restricción, incluso, los termoplásticos pueden no ser reciclados si están tan contaminados, que es más costoso limpiarlos que botarlos.

El mercado del reciclaje no procesa material reciclable cuando es poco atractivo económicamente, ya que los diferentes tipos de plásticos requieren un conocimiento especializado para su correcta separación. Además, la variedad de plásticos no permite obtener cantidades significativas de algunos tipos, por lo que no se pueden reducir los costos de procesamiento por unidad, haciendo no rentable el reciclaje de ciertos plásticos

A nivel mundial, la industria ha desarrollado diferentes tecnologías y alternativas para que el plástico acelere su proceso de degradación y se biodegraden. La biodegradación consiste en degradar un material con hongos, bacterias y otros microorganismos, para obtener dióxido de carbono, metano, compuestos inorgánicos, agua y biomasa.

Los polímeros se convierten en biodegradables en ambientes aerobios, como el compostaje o en el agua o, en ambientes anaerobios, como en los rellenos sanitarios. En condiciones aerobias, el carbono es oxidado biológicamente a dióxido de carbono, liberando energía, que es aprovechada por los microorganismos. Bajo condiciones anaerobias, se produce $\mathrm{CO}_{2}$ más metano. La biodegradación que se da en los rellenos genera emisiones de metano que contribuyen al cambio climático (BPI, 2010; Platt, s.f).

Los productos biodegradables más conocidos son los oxobiodegradables. La biodegradación de estos consiste en la introducción de metales (cobalto, manganeso, magnesio, 
hierro, zinc, entre otros), que en teoría fomenta la oxidación y rompe la cadena del plástico, cuando se expone al calor, aire o luz. Al romper la cadena, los microorganismos encontrados en los sitios de disposición final se alimentarían del material. Para los rellenos, no se ha presentado datos que soporten una completa biodegradación en los ambientes anaeróbicos. En condiciones climáticas áridas con exposición al calor y a la luz solar, los aditivos oxo-biodegradables aceleran la fragmentación tradicional de los polímeros; sin embargo, la fragmentación no es señal de biodegradación y no se registran datos que muestren por cuánto tiempo los fragmentos se mantienen en los suelos o en el agua (BPI, 2010).

Existen métodos para medir la descomposición, estandarizados en Estados Unidos. La Sociedad Americana de Pruebas y Materiales (ASTM, American Society of Testing and Materials) definió qué constituye la biodegradabilidad, de acuerdo a los ambientes de disposición final. En Europa y en otros países tienen estándares similares, según sus condiciones propias. En Colombia, no existen estos estándares y no actúa ningún organismo que compruebe que los productos que se venden en el mercado local efectivamente cumplan con la biodegradación en las condiciones locales. En Estados Unidos, la División Nacional de Publicidad del Consejo de Mejores Negocios, cuyo objetivo es comprobar la veracidad de la publicidad, recomendó descontinuar las bolsas que aseguran ser oxo-degradables, por no cumplir con los estándares, demostrado en California, por lo tanto, en este Estado, se restringió el uso de los términos "compostable", "biodegradable", "degradable" y "degradable en agua" en las bolsas plásticas (Platt, s.f.). Incluso, el término "reciclable" estaría no sustentado, pues no se ha comprobado que los aditivos para la biodegradación no afecten el proceso de reciclaje.

De esta revisión, se concluye que es necesario realizar investigaciones tendientes a evaluar, en nuestro medio, prácticas, ante todo de acolchado, utilizadas en países con un mayor desarrollo agrícola y, además, seguir trabajando en la búsqueda de polímeros biodegradables, tanto para fines agrícolas como para otros usos. Igualmente, se puede indicar que dado el beneficio que el uso del plástico representa, no más en el ahorro de agua de riego y de herbicidas, existe un equilibrio entre beneficio y costo ambiental.

Conflicto de interés: El manuscrito fue preparado y revisado por los autores, quienes declaramos que no existe ningún conflicto de intereses que ponga en riesgo la validez de los resultados presentados.

\section{BIBLIOGRAFÍA}

1. ADENTUJI, I. 1993. Effect of mulches and irrgation on growth and yield of lettuce in semi-arid región. Hort. Abstracts. 63:1147.
2. AL-ASSIR, I.; RUBEIZ, I.; KHOURY, R. 1992. Response of all greenhouse cos lettuce to clear mulch and nitrogen fertilizer. Hort. Abstracts. 62:3893.

3. ARRUDA, F.P.; ANDRADE, A.P.; BELTRÃO, N.E; PEREIRA, W.E.; LIMA, J.R.F. 2005. Viabilidade econômica de sistemas de preparo do solo e métodos de controle de Tiririca em algodoneiro. Rev. Bras. Eng. Agríc. Ambient. 9(4):481-488.

4. ASOC. CIUDADES Y REGIONES PARA EL RECICLAJE-ACRR, ASOC. FABRICANTES DE PLÁSTICOS DE EUROPA-APME, CONSEJO EUROPEO FABRICANTES DE VINILIO-ECVM, RECICLADORES EUROPEOS DE PLÁSTICOS- EUPR, TRANSFORMADORES EUROPEOS DE PLÁSTICOS-EUPC. 2004. Guía de Buenas Prácticas para el Reciclaje de los Residuos Plásticos. Una guía por y para las autoridades locales y regionales. s.p.

5. BANACOL. s.f. Fábrica de plásticos. Disponible desde Internet en: www.banacol.com/Fabricaplasticos/359/1/ FabricadePlasticos (con acceso 23/07/2012).

6. BARALDI DE PAULI, R.; BATTESTIN QUAST, L.; MOTTIN DEMIATE, I.; SETSUKO SAKANAKA, L. 2011. Production and characterization of oxidized cassava starch (Manihot esculenta Crantz) biodegradable films. Stärke (Starch). 63(10):595-603.

7. BARNES, D.A.; GALGANI, F.; THOMSON, R.C.; BARLAZ, M. 2009. Accumulation and fragmentation of plastic debris in global environments. Philos Trans $\mathrm{R}$ Soc Lond B Biol Sci. 364(1526):1985-1998.

8. BARRERA, J.L.; VERGARA, D.C.; MARÍN, O.J. 2007. Contribución del desmane y embolse del racimo a la producción y calidad del plátano hartón. Agron. 15(1):39-44.

9. BELALCAZAR C., S.; SALAZAR M., C.A.; CAYÓN S., G.; LOZADA Z., J.E.; CASTILLO, L.E.; VALENCIA M., J.A. 1991. Manejo de Plantaciones. En. Belalcazar C. (ed.), El Cultivo del Plátano en el Tropico. ICA. Man. Asist. Técn. 50. Impr. Feriva Ltda. Cali. 376p.

10. BERRILL, F.W. 1956. Bunch covers for bananas. Queensland Agr. J. 82(8):435-440.

11. BIODEGRADABLE PRODUCTS INSTITUTE BPI. 2010. Background on Biodegradable Additives. Disponible desde Internet en: http://www.bpiworld.org/resources/Documents/Biodegradable\%20Additives\%20Fa ct\%20Sheet\%20v8\%20July\%2009.pdf (con acceso 3/03/2013). 
12. BRINGHURST, R.; VOTH, V. 1990. Culture and physiological manipulation of California strawberries. Hortscience 25 (8):889-892.

13. CHAVARRIA, G.; SANTOS, H. P. dos. 2009. Manejo de videiras sob cultivo protegido. Cienc. Rural [online]. 39(6):1917-1924.

14. CHAVARRIA, G.; SANTOS, H.P. dos; SÔNEGO, O.R.; BETTIO MARODIN, G.A.; BERGAMASCHI, H.; CARDOSO, L.S. 2007. Incidência de doenças e necessidade de controle em cultivo protegido de videira. Rev. Bras. Frutic. 29(3):477-482.

15. CONTRERAS, A.; BORIE, F.; RUBIO, R.; MORAGA, E. 1993. The effects of plastic protection on the development of mycorrhizal infection in roots of diferent horticultural cultivars. Hort. Abstracts 63:1117.

16. CONVERSE, R. 1981. The Israeli strawberry industry. Hortscience. 16(1):19-22.

17. COSTA, J.N.M.; SCARPARE FILHO, J.A.; KLUGE, R.A. 2002. Efeito do ensacamento de cachos de banana 'Nanicão' na produção e no intervalo entre inflorescência e colheita. Pesq. Agropec. Bras. 37(11):15751580 .

18. DELGADO, A.E.; APERADOR, W.; BAUTISTA RUIZ, J.H. 2011. Optical properties of Idpe films with different additives mixtures. Ing. Cienc. [online]. 7(14):49-70.

19. EICHIN, R.; DEISER, E. 1991. Paper mulch in cabbage lettuce. Hort. Abstracts 61: 3675.

20. ESPI, E.; SALMERÓN, A.; FONTECHA, A.; GARCÍA, Y.; REAL, A.I. 2006. PLastic films for agricultural applications. J. Plastic Film Sheeting. 22(2):85-102.

21. FAO. 2007. Faostat Estadística Databases, Agricultura, Cultivos Primarios, Tomate. Disponible desde Internet en: http://Faostat. Fao.Org/Site/340/Default.Aspx (con acceso 20(10/12).

22. GÓMEZ, P.; ROMERO, F. 2002. Evaluación del insecticida piretroide Bifentrina impregnado en la funda para el control de plagas del racimo en el cultivo de banano en Machala, Ecuador. (Musa paradisíaca L). En: Memorias XV Reunión Asociación de Bananeros de Colombia AUGURA. Cartagena de Indias. 27 de Octubre - 2 de Noviembre 2002.p.202-206.

23. GONZÁLEZ-VIÑAS, W.; MANCINI, H.L. 2003. Ciencia de los Materiales. Editorial Ariel Ciencia (España). 240p.
24. HISKAKIS, M.; BRIASSOULIS, D.; BABOU, E.; LIANTZAS, K. 2008. Agricultural plastic waste mapping in Greece. Acta Hort. (ISHS) 801:351-358.

25. LIMA, M.B. 2002. O ensacamento do cacho da bananeira. Cruz das Almas-BA. Embrapa Mandioca e Fruticultura. 2p.

26. LEFF, E. 2003. Ecología y Capital. Siglo XXI Editores, España. 437p.

27. MACÍAS, H.; MUÑOZ, J.A.; VELÁSQUEZ, M.A.; SÁNCHEZ, I. 2011. Tecnología de producción de plántula y cosecha de chile con plasticultura. Caso de estudio: Región Lagunera. En: Spring, O. (ed.) Los retos de la investigación del agua en México. UNAM. p.283-290.

28. MEDEIROS, J.F. de; SILVA, M.C. de C.; CÂMARA NETO, F.G.; ALMEIDA, H.B. de ; O. SOUZA, J. de; NEGREIROS, M.Z. de; SOARES, S.P.F. 2006. Crescimento e produção do melão cultivado sob cobertura de solo e diferentes freqüências de irrigação. Rev. Bras. Eng. Agríc. Ambient. [online]. 10(4):792-797.

29. MEDINA, J.A.; SALAS, J.C. 2003. Caracterización morfológica del granulo de almidón nativo: Apariencia, forma, tamaño y su distribución. Rev. Ing. [online]. 27:56-62.

30. MEDINA, A.; COOMAN, A.; PARRADO, C.A; SCHREVENS, E. 2006. Evaluation of energy use and some environmental impacts for greenhouse tomato production in the high altitude tropics. Acta Hort. (ISHS) 718:415-422.

31. MENESES, J.; CORRALES, C.M.; VALENCIA, M. 2007. Síntesis y caracterización de un polímero biodegradable a partir del almidón de yuca. Rev. EIA. Esc. Ing. Antioq. [online]. 8:57-67.

32. MOREIRA, A. 2008. Proteção de cachos de bananeira com sacos de polietileno nas condições edafoclimáticas do estado do Amazonas. Ciênc. Agrotec. 32(1):129-136.

33. MOREIRA, R.S. 1987. Banana: teoría e práctica de cultivo. Campinas; Fundação Cargill. 335p.

34. OLEFINAS PLÁSTICOS PARA EL DESARROLLO. s.f. Fundas para embolsado (treebags). Disponible desde Internet en: www.olefinas.com/index.php/es/bananero/treebags (con acceso 23/07/2012). 
35. ORIOLANI, M.; DE TORRONTEGUI, A.; MARTIN, G. 1979. Uso de película de polietileno como cobertura de suelo. Folleto INTA No 57. 30p.

36. PLATT, B. (s.f.). Biodegradable Plastics: True or False? Good or Bad? Disponible desde Internet en: http:// www.sustainableplastics.org/spotlight/biodegradable-plastics-true-or-false-good-or-bad (con acceso 15/10/2012).

37. RENQUIST, T.A.; MARTIN, L.W.; BREEN, P.J. 1982. Effect of polyethylene mulch and summer irrigation regimes on subsequent flowering and fruiting of "Olympus" strawberry. J. Am. Soc. Hort. Sci. 107(2):373-376.

38. RESOLUCIÓN NÚMERO (0272) 16 de febrero 2009. Por la cual se acepta un plan de gestión de devolución de productos posconsumo de plaguicidas" Ministerio de Ambiente, Vivienda y Desarrollo Territorial. Disponible desde Internet en: www.minambiente. gov.co/documentos/res_0272_160209.pdf (con acceso 12/05/2012).

39. RESOLUCIÓN 637 DE 2004. Licencia Ambiental para la disposición final de desechos y/o sustancias peligrosas. Disponible desde Internet en: www.avancejuridico.com/actualidad/.../r_mavdt_0637_2004.html (con acceso 12/05/2012).

40. RODRIGUES, M.G.V.; SOUTO, R.F.; MENEGUCCI, J.L.P. 2001. Influência do ensacamento do cacho na produção de frutos da bananeira-'prata-anã' irrigada, na região norte de minas gerais. Rev. Bras. Frutic. 23(3):559-562.

41. ROBINSON, J.C.; NEL, D.J. 1984. Banana bunchs covers in winter. Citrus Subtrop. Fruit Res. 138(1):5-6.

42. ROBINSON, J.C. 1996. Bananas and Plantains.CAB International, Wellingford, WK. 238p.

43. RUIZ, G.; MONTOYA, C.; PANIAGUA, M. 2009. Degradabilidad de un polímero de almidón de yuca. Rev. EIA. 12:67-78.

44. RUIZ AVILES, G. 2006. Obtención y caracterización de un polímero biodegradable a partir de almidón de yuca. Ing. y Ciencia. 2(4):5-28.

45. SANTOS, B.M.; OBREGÓN, H.A. 2009. Prácticas culturales para la producción comercial de fresas en Florida. Depto. Horticultural Sci., Servicio de Extensión Cooperativa de la Florida. U. de Florida. 15p.
46. SANZ, D. 2012. Plástico fabricado con maíz para el cultivo de la fresa. Disponible desde Internet en: www. ecologiaverde.com/plastico-fabricado-con-maiz-para-el-cultivo-de-la-fresa/ (con acceso 11/05/2012).

47. SCHREVENS, E.; PORTIER, K.; DARIUS, P.; COOMAN, A.; TENORIO, T.; MEDINA, A. 2005. Simulation-based environments for practicing data-collection skills in greenhouse experimentation. Acta Hort. 691:871876.

48. SCIVITTARO, W.B.; MELO, A.M.T.; TAVARES, M.; AZEVEDO FILHO, J.A. de; CARVALHO, C.R.L.; RAMOS, M.T.B. 1999. Caracterização de híbridos de pimentão em cultivo protegido. Hort. Bras. 17(2):147-150.

49. SILVA, M.C. de C.; MEDEIROS, J.F. de; NEGREIROS, M.Z. de; SOUSA, V.F. de. 2005. Produtividade de frutos do meloeiro sob diferentes níveis de salinidade da água de irrigação, com e sem cobertura do solo. Hort. Bras. [online]. 23(2):202-205.

50. STAVISKY, A. 2010. Plasticultura en la Argentina. Informe Frutihortícola. Comité Arg. Plásticos para la Producción Agropecuaria, CAPPA. 4p.

51. STEVENS, E.S. 2001. Green Plastics: An introduction to the New Science of Biodegradable Plastics. Princetown University Press. 238p.

52. TEUTEN, E.L.; SAQUING, J.M.; KNAPPE, D.R.U.; BARLAZ, M.A.; JONSSON, S.; BJÖRN,A.; ROWLAND,S.J.; THOMPSON,R.C.; GALLOWAY, T.S.; YAMASHITA,R.; OCHI, D.; WATANUKI, Y.; MOORE, C.; VIET, P.H.; TANA, T.S.; PRUDENTE, M.; BOONYATUMANOND, R.; ZAKARIA, M.P.; AKKHAVONG, K.; OGATA, Y.; HIRAI, H.; IWASA, S.; MIZUKAWA, K.; HAGINO, Y.; IMAMURA, A.; SAHA, M.; TAKADA, H. 2009. Transport and release of chemicals from plastics tothe environment and to wildlife. Phil. Trans. R. Soc. B: Biol. Sci. 364: 2027-2045.

53. THORNTON, J. 2002. Environmental impacts of polyvinyl chloride building materials - A Healthy Building Network report. Washington, DC: Healthy Building Network. Disponible desde Internet enhttp://www. healthybuilding.net/pvc/Thornton_Enviro_Impacts_ of_PVC.pdf (con acceso 21/10/2012).

54. THOMPSON, R.C.; SWAN, S.H.; MOORE, C.J.; SAAL, F.V. 2009. Our plastic age. Phil. Trans. R. Soc. B. 364:1973-1976. 
55. THOMPSON, R.C.; OLSEN, Y.; MITCHELL, R.P.; DAVIS, A.; ROWLAND, S.J.; JOHN, A.W.G.; MCGONIGLE, D.; RUSSELL, A.E. 2004. Lost At Sea: Where is All the Plastic? Science. 304:838.

56. TRINIDADE, A.V.; BORGES, A.N.; TEXEIRA, A.H.; MATOS, A.; RITZINGER, C.H.; ALMEIDA, C.; COSTA, D.; DA COSTA, E.L; ALVES, E.J.; COELHO, E.; MATSUURA, F.C.; DOS SANTOS-SEREJO, J.; DE CARVALHO, J.E.; SOUZA, J.; SOUZA, L.; SANTANA, M.; LIMA, M.; PEREIRA, M.; FRANCELLI, M.; FOLEGATTI, M.I.; MEISSNER FILHO, P.E.; DE OLIVEIRA E SILVA,S.; MEDINA V.; CORDEIRO, Z.J. 2004. Ensacamento do Cacho. En: Borges, A.L.; da Silva Souza (eds). O Cultivo da Bananeira. Embrapa. Cruz das Almas-BA. p.121-123.

57. VARGAS-CALVO, A.; VALLE-RUIZ, H. 2011. Efecto de dos tipos de fundas sobre el fruto de banano (Musa AAA). Agron. Mesoam. 22(1):81-89.
58. VARGAS, A.; VALLE, H.; GONZÁLEZ, M. 2010. Efecto del color y de la densidad del polietileno de fundas para cubrir el racimo sobre dimensiones, presentación y calidad poscosecha de frutos de banano y plátano. Agron. Costarricense. 34(2):269-285.

59. VIDA, J.B.; ZAMBOLIM, L.; TESSMANN, D.J.; BRANDÃO Filho, J.U.T.; VERZIGNASSSI, J.R.; CAIXETA, M.P. 2004. Manejo de doenças de plantas em cultivo protegido. Fitopatol. Bras. [online]. 29(4):355-372.

60. YURI, J.E.; RESENDE, G.M de; COSTA, N.D.; MOTA, J.H. 2012. Cultivo de morangueiro sob diferentes tipos de mulching. Hortic. Bras. [online]. 30(3):424427.

Recibido: Mayo 25 de 2012

Aceptado: Abril 3 de 2013 